\title{
Quality of Life Is More Impaired in Patients Seeking Medical Care for Food Allergy
}

\author{
Thuy-My Le ${ }^{a}$ Bertine M.J. Flokstra-de Blok ${ }^{b} d$ Els van Hoffen ${ }^{a}$ Ans F.M. Lebens ${ }^{a}$ \\ Nicole J. Goossens ${ }^{c, d}$ Anthony E.J. Dubois ${ }^{c, d}$ Carla A.F.M. Bruijnzeel-Koomen ${ }^{a}$ \\ André C. Knulst ${ }^{\mathrm{a}}$ \\ ${ }^{a}$ Department of Dermatology and Allergology, University Medical Center Utrecht, Utrecht, ${ }^{b}$ Department of General \\ Practice, ${ }^{C}$ Department of Paediatrics, Division of Paediatric Allergy and Paediatric Pulmonology and ${ }^{\mathrm{d}}$ GRIAC Research \\ Institute, University Medical Center Groningen, University of Groningen, Groningen, The Netherlands
}

\section{Key Words \\ Food allergy · Quality of life}

\begin{abstract}
Background: Food allergy (FA) affects $2-4 \%$ of adults, but only a small percentage visit an outpatient clinic for a thorough evaluation. Methods: A matched case-control study was used to compare health-related quality of life (HRQL) of the Dutch general population that did not seek medical care for their FA with outpatients who did seek medical care. All participants were diagnosed as food allergic (i.e. with a suggestive history and corresponding positive $\operatorname{lgE}$ ). HRQL was measured using the Food Allergy Quality of Life Questionnaire - Adult Form (FAQLQ-AF). A food allergy independent measure (FAIM) was used to evaluate the adult's perception of the severity of his/her disease. Results: Total FAQLQ-AF score in individuals who never visited a doctor for their FA was significantly lower than that of patients who sought medical care ( 2.4 vs. $3.9, p=0.03$ ), indicating that the former had a better quality of life than patients who did seek medical care. Regarding the different domains of FAQLQ, the score for allergen avoidance and dietary restrictions and the score for emotional impact (EI) was significantly higher in the group that sought medical care $(p=0.02$ and 0.03 , respec-
\end{abstract}

tively), indicating the importance of these domains. The FAIM score was significantly higher in the group that sought medical care, indicating that they perceived their FA as more severe. Conclusion and Clinical Relevance: Patients who seek medical care for their FA have a more impaired $\mathrm{HRQL}$ and perceive their FA as more severe. Food avoidance and issues related to the El of FA are key areas of intervention aimed at improving $\mathrm{HRQL}$ in patients with FA.

(C) 2013 S. Karger AG, Basel

\section{Introduction}

Food allergy (FA) is estimated to affect $2-4 \%$ of adults [1]. Severe food-allergic reactions can be potentially life threatening. Therefore, patients with severe reactions should be carefully evaluated by a physician and advised with respect to avoidance of the offending food and how and when to use an epinephrine auto-injector. We previously showed that $52 \%$ of patients with severe food-allergic reactions do not seek medical care, whereas $44 \%$ of patients with only mild symptoms do seek medical care [2]. It is unknown why some patients seek medical care for their food-allergic reactions, whilst others do not. A possible explanation is a difference in health-related qual-

\section{KARGER}

E-Mail karger@karger.com

www.karger.com/iaa
(C) 2013 S. Karger AG, Basel

$1018-2438 / 13 / 1624-0335 \$ 38.00 / 0$
Correspondence to: Dr. Thuy-My Le

Department of Dermatology and Allergology (G02.124)

University Medical Center Utrecht

PO Box 85500, NL-3508 Utrecht (The Netherlands)

E-Mail t.t.m.le-2@umcutrecht.nl 
Fig. 1. Flow-diagram of the epidemiological part of the EuroPrevall study in the general population. \# Priority food = Hen's egg, cow's milk, peanut, soy, hazelnut, walnut, celery, kiwi, apple, peach, sesame, mustard, wheat, fish and shrimp, buckwheat, corn, carrot, tomato, melon, banana, lentils, sunflower, poppy seeds. ${ }^{\ddagger}$ FAQLQ was filled in before clinical evaluation of FA by a doctor because clinical evaluation could influence FAQL. ${ }^{*}$ Not eligible anymore because the participant was already included and clinically evaluated for FA by a doctor as part of the EuroPrevall study.

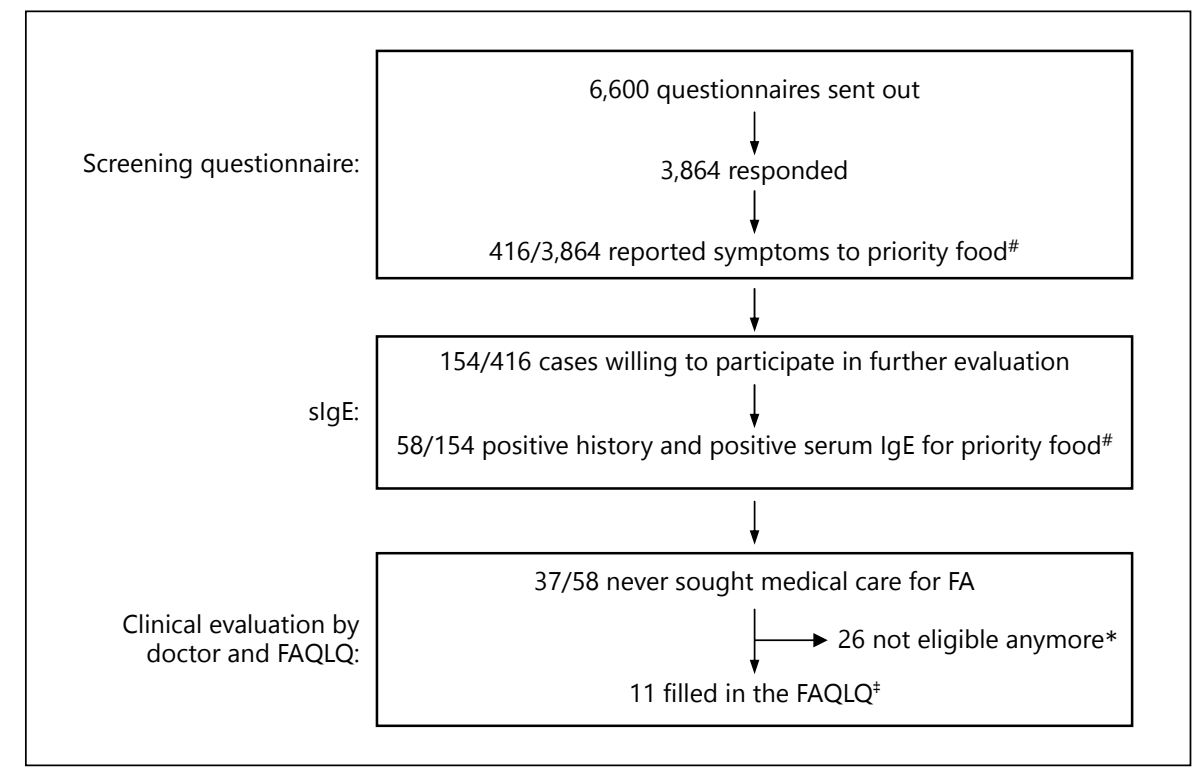

ity of life (HRQL). Various studies have shown that FA has a negative impact on an individual's HRQL [3-5]. A more impaired HRQL could be a reason to seek medical care, irrespective of the severity of symptoms. Therefore, the aim of this study was to investigate the HRQL in patients that did or did not seek medical care for their FA.

\section{Methods}

\section{Participants}

A matched case-control study design was used to compare the HRQL from the Dutch general population that did not seek medical care for their FA with outpatients that did seek medical care. Both groups participated in the EuroPrevall project, a multidisciplinary European study on several aspects of FA [6]. The epidemiological part of the study has been described in detail elsewhere [7]. Figure 1 shows the flow-diagram for the epidemiological part of the EuroPrevall study that was conducted in the Netherlands. In short, a screening questionnaire was sent out to a random sample of 6,600 adults of a general Dutch urban population (Utrecht City). Of the 3,864 responders, 416 had symptoms of preselected priority foods, which are foods that were expected to cause most of the food allergic reactions. Of these 416, 154 were willing to come to our clinic for sIgE testing. Fifty-eight of the 154 tested participants $(38 \%)$ had a suggestive history of FA and a corresponding positive sIgE. These 58 participants were invited to come to our tertiary center for a clinical evaluation (thorough clinical history, skin-prick test and if necessary additional sIgE testing) by a medical doctor. The clinical evaluation also included an explanation about FA, advice on food avoidance and prescription of an epinephrine auto-injector when appropriate. Thirtyseven out of these 58 individuals had never sought medical care for their FA (i.e. had never visited a doctor for evaluation and advice about their FA, and had never been diagnosed as food allergic by a doctor).

The plan to administer a disease-specific HRQL questionnaire arose during the latter part of the project. Therefore, of the 37 participants that had never sought medical care for their FA, only the last 11 individuals were asked to participate in this study. This consisted of filling in a HRQL questionnaire before the clinical evaluation by a doctor in our tertiary center. Since clinical evaluation could influence HRQL, it was important that participants filled in the questionnaire prior to the clinical evaluation. Twenty-six of the 37 participants that had never sought medical care for their FA were not eligible because they had already been clinically evaluated by a doctor in our center as part of the Europrevall study. The response rate of the remaining 11 participants was $100 \%$. These 11 individuals were individually matched with 11 outpatients who were referred to our outpatient clinic with a suspicion of FA and who took part in the outpatient clinical part of the EuroPrevall study [6]. Diagnosis of FA, in the general population as well as in the outpatients, was based on a convincing history (i.e. symptoms within $2 \mathrm{~h}$ of ingestion of a food) together with a positive $\operatorname{sigE}$ $\left(\geq 0.35 \mathrm{kU}_{\mathrm{A}} / \mathrm{l}\right)$ for that food. Since a previous study showed that patients with a severe FA (i.e. cardiovascular symptoms) and patients who reported more than three food allergies had a greater perceived impairment of HRQL [8], the highest priority was matching based on these two variables. In addition, we matched for sex, age and type of food.

This study was approved by the local ethical committee of the University Medical Center Utrecht. All participants signed informed consent prior to inclusion.

\section{Questionnaire}

HRQL was measured using the Food Allergy Quality of Life Questionnaire - Adult Form (FAQLQ-AF), a validated disease-specific HRQL questionnaire, consisting of 29 questions [8]. A food allergy independent measure (FAIM), consisting of 6 items, measured the adult's perception of the severity of his/her condition [8]. Both 
questionnaires used a 7-point scale, with a higher FAQLQ-AF score indicating a more impaired HRQL and a higher FAIM score indicating a more severe perceived FA. The total FAQLQ-AF score and FAIM score was the mean score of all items. Total FAQLQ-AF and FAIM scores were compared between individually matched cases (i.e. food-allergic individuals from the general population who had never sought medical care for their FA) and controls (food-allergic outpatients who had sought medical care for their FA). Two years after the FAQLQ-AF and FAIM were completed, we asked the participants to complete the questionnaires again to investigate whether the visit to our allergy center for an evaluation and explanation of the FA and doctor's advice had changed the HRQL.

\section{Clinical Severity}

The most severe food-allergic reaction was classified according to a modified Mueller classification $[9,10]$. Symptoms involving the oral cavity were classified as Mueller grade 0 , symptoms of the skin and mucous membranes as grade 1, gastro-intestinal symptoms as grade 2, respiratory symptoms as grade 3 and cardiovascular symptoms as grade 4 [10].

\section{Statistical Analysis}

Differences in FAQLQ-AF and FAIM score between participants that did and did not seek medical care and between the 1st and 2nd questionnaire were tested using the paired Wilcoxon signed-rank test. Statistical analysis was performed using SPSS version 15 (2007; SPSS Inc., Chicago, Ill., USA) for Windows.

\section{Results}

Patient characteristics are shown in table 1. Both groups were comparable with regard to the severity of the reaction and number of foods involved: in the group that never visited a doctor for their FA there was 1 more individual with a Mueller 0 reaction (i.e. only oral allergy symptoms) and 1 more with reactions to more than three foods compared to the other group (table 1). The time between clinical reaction and HRQL investigation did not differ significantly between both groups $(\mathrm{p}=0.31)$.

The differences in total FAQLQ-AF score, FAQLQ domains and the total FAIM score are presented in table 2. Total FAQLQ-AF score in individuals who visited a doctor for their FA was significantly higher than that of patients who did not seek medical care (mean \pm SD: $3.9 \pm$ 1.7 vs. $2.4 \pm 0.9, \mathrm{p}=0.03$ ), indicating that the former had a poorer quality of life. A previous study demonstrated that the minimal important difference of HRQL questionnaires using a 7-point scale, i.e. the smallest difference in total FAQLQ-AF scores which patients perceive as beneficial, is approximately 0.5 [11]. The difference in total FAQLQ-AF scores between both groups in our study was 1.5. Therefore, the difference in FAQLQ-AF score was not only statistically, but also clinically significant.
Table 1. Patient characteristics

\begin{tabular}{llll}
\hline Sex & $\begin{array}{l}\text { Age, } \\
\text { years }\end{array}$ & $\begin{array}{l}\text { Severity } \\
\text { (Mueller- } \\
\text { classification) }\end{array}$ & $\begin{array}{l}\text { Foods, Foods } \\
\mathrm{n}\end{array}$ \\
\hline
\end{tabular}

Never visited a doctor for symptoms of FA

$\begin{array}{lllll}\text { Female } & 23 & 0 & \leq 3 & \text { FR, VG } \\ \text { Female } & 39 & 1 & \leq 3 & \text { SF } \\ \text { Female } & 29 & 1 & \leq 3 & \text { TN, FR } \\ \text { Female } & 44 & 3 & \leq 3 & \text { PN, MI } \\ \text { Female } & 27 & 3 & >3 & \text { PN, TN, FR } \\ \text { Female } & 40 & 3 & \leq 3 & \text { TN, FR } \\ \text { Male } & 41 & 3 & \leq 3 & \text { FR } \\ \text { Female } & 30 & 3 & \leq 3 & \text { TN, FR } \\ \text { Female } & 32 & 3 & >3 & \text { PN, TN, FR } \\ \text { Female } & 36 & 3 & \leq 3 & \text { TN, FR } \\ \text { Female } & 51 & 4 & >3 & \text { PN, TN, FR }\end{array}$

Visited a doctor for symptoms of FA

$\begin{array}{lllll}\text { Female } & 35 & 1 & \leq 3 & \text { FR, SF } \\ \text { Female } & 39 & 1 & \leq 3 & \text { TN, FR } \\ \text { Male } & 33 & 1 & \leq 3 & \text { TN, FR, VG } \\ \text { Female } & 20 & 3 & \leq 3 & \text { PN, FR } \\ \text { Female } & 24 & 3 & \leq 3 & \text { PN, TN, FR } \\ \text { Female } & 39 & 3 & \leq 3 & \text { TN, FR } \\ \text { Male } & 33 & 3 & \leq 3 & \text { FR } \\ \text { Female } & 28 & 3 & \leq 3 & \text { TN, FR } \\ \text { Female } & 58 & 3 & >3 & \text { TN, EG, FR } \\ \text { Male } & 36 & 3 & \leq 3 & \text { TN, FR } \\ \text { Female } & 47 & 4 & >3 & \text { PN, TN, FR, MI }\end{array}$

$\mathrm{EG}=$ Egg; FR = fruit; $\mathrm{PN}=$ peanut $\mathrm{TN}=$ tree nuts; $\mathrm{SH}=$ shell fish; $\mathrm{VG}=$ vegetables; $\mathrm{MI}=$ milk .

${ }^{1}$ Classification of the most severe reaction using a modified Mueller classification: 0 = oral allergy symptoms, $1=$ skin/mucous membrane symptoms, 2 = gastro-intestinal symptoms, 3 = respiratory symptoms, $4=$ cardiovascular symptoms.

Table 2. FAQL (domains) and FAIM scores

\begin{tabular}{llll}
\hline & $\begin{array}{l}\text { Never visited } \\
\text { a doctor } \\
\text { for FA }\end{array}$ & $\begin{array}{l}\text { Visited a } \\
\text { doctor } \\
\text { for FA }\end{array}$ & $\begin{array}{l}\mathrm{p} \\
\text { value }\end{array}$ \\
\hline FAQL & $2.4 \pm 0.9$ & $3.9 \pm 1.7$ & 0.03 \\
$\begin{array}{l}\text { Domains of FAQL } \\
\text { AADR }\end{array}$ & $2.2 \pm 0.9$ & $4.0 \pm 1.8$ & 0.02 \\
EI & $2.4 \pm 1.0$ & $3.8 \pm 1.8$ & 0.03 \\
RAE & $2.4 \pm 1.4$ & $3.6 \pm 1.9$ & 0.13 \\
FAH & $3.0 \pm 1.5$ & $4.3 \pm 1.8$ & 0.11 \\
FAIM & $2.4 \pm 0.7$ & $3.6 \pm 1.4$ & 0.005 \\
\hline
\end{tabular}

Values are mean \pm SD. RAE $=$ Risk of accidental exposure; $\mathrm{FAH}=$ food allergy-related health. 
Regarding the different domains of FAQLQ, only the score for allergen avoidance and dietary restrictions (AADR) and for emotional impact (EI) was significantly higher in the group that sought medical care, indicating that the impaired quality of life in the group that sought medical care can be largely attributed to these two domains. In the domain of AADR, patients who sought medical care found it significantly more troublesome that they were restricted in the number of products they could eat $(\mathrm{p}=0.02)$, were limited as to the products they could buy $(p=0.03)$, had to read labels $(p=0.03)$, had to refuse many products during social activities $(\mathrm{p}=0.02)$, were less able to taste or try products when eating out $(\mathrm{p}=$ 0.01 ), had to personally check whether they could eat something when eating out $(p=0.01)$, hesitated eating a product when they had doubts about it $(\mathrm{p}=0.02)$ and had to explain to those around that they have a food allergy $(p=0.05)$. In the domain of EI, patients who sought medical care were more frightened of a food-allergic reaction $(p=0.03)$, more frightened of accidentally eating the wrong food $(p=0.005)$ and more frightened of an allergic reaction when eating out despite the fact that dietary restrictions had been discussed beforehand $(p=0.04)$.

The clinical classification of severity using the adapted Mueller classification [10] did not differ between the groups. Remarkably, however, the FAIM score was higher in the group that sought medical care $(\mathrm{p}=0.005)$, indicating that they perceived their FA as being more severe.

Two years after the FAQLQ-AF and FAIM were completed, we asked the participants to complete the questionnaires again. The questionnaire was returned by 9 out of 11 individuals who never sought medical care and 10 out of 11 patients that sought medical care. No differences in FAQLQ scores and FAIM scores were seen between the first and the second measurement in the group that never sought medical care (FAQLQ-AF score, mean \pm SD: $2.4 \pm 0.9$ vs. $2.2 \pm 1.0, p=0.77$, and FAIM score: $2.4 \pm 0.7$ vs. $2.3 \pm 0.9, p=0.26$ ), as well as in the group that sought medical care (FAQLQ-AF score, mean \pm SD: $3.9 \pm 1.7$ vs. $3.9 \pm 1.8, \mathrm{p}=0.41$ and FAIM score: $3.6 \pm 1.4$ vs. $3.5 \pm 1.6, \mathrm{p}=0.77)$.

\section{Discussion}

Various studies have shown that FA has a negative impact on an individual's HRQL [3-5]. Within the framework of the EuroPrevall project, an FA-specific quality of life questionnaire for adults has been developed and vali- dated, which was used in this study [8, 12]. The study shows that although the severity of the food-allergic reaction was similar between participants that did and did not seek medical care, HRQL was significantly more impaired in patients who did seek medical care. This indicates that a poorer HRQL may be an important reason for patients to seek medical care for FA. Previous studies have shown that around half of patients with a severe food-allergic reaction do not visit a doctor for evaluation and advice [2]. In this study we showed that a less impaired HRQL could be a reason for not seeking medical care despite severe food-allergic symptoms. Patients that do not seek medical care are, for example, less frightened of a foodallergic reaction and experience the dietary restrictions and food avoidance as less troublesome, which could be an explanation for not seeking medical care. However, it is important that patients with severe food-allergic reactions (i.e. respiratory or cardiovascular symptoms) do visit a physician for evaluation of their FA and advice on food avoidance, and also for prescription for and instruction in the use of an epinephrine auto-injector. It has been shown that fatal anaphylaxis is associated with failure or delay in the administration of epinephrine [13].

Participants filled in the questionnaires twice: the first questionnaire was completed before a full clinical evaluation in our center was performed, and the second 2 years after that. The unchanged HRQL in the group that did as well as the group that did not seek medical care indicates that the visit to our clinic with a full clinical evaluation and explanation of FA and its treatment options by a doctor did not influence the HRQL of these patients. In patients that seek medical care, the two domains of AADR and EI differed significantly compared to patients that did not seek medical care, indicating that the impaired quality of life in the group that sought medical care can be attributed to these domains. To date, the only therapy for FA is strict avoidance of the offending allergen, which is difficult due to hidden allergens. Our data suggest that the effort required to avoid accidental exposures (especially when eating out and during social activities) and the inherent uncertainty of success impairs the HRQL of patients and may be the reason for seeking medical care. To improve the HRQL of food-allergic patients, more attention should be paid to advice on dietary restrictions and how to avoid accidental exposures. Furthermore, the EI of FA for the patient should be addressed and evaluated.

This study has some limitations. The study consisted of a small sample size. However, selection bias is unlikely because the response rate of those who received the questionnaire was $100 \%$ and the matched cases and controls 
were largely comparable. Despite the small number of participants, a large and significant difference in HRQL scores was observed which exceeded the minimal important difference. In this study, matching of cases and controls was mainly based on clinical severity and the number of foods that caused a reaction, because a previous study showed that these factors influence HRQL [8]. Although we also tried to match on gender, the group that never had sought medical care consisted of two more females. Previous studies have shown that the perception of HRQL in women is in general worse than in men $[14,15]$. Therefore, we expect that if both groups in our study were similar regarding gender, the difference found in HRQL between both groups would be even greater. Other factors that were not measured in this study (such as socioeconomic and sociodemographic state) may also have influenced HRQL.

In conclusion, patients that seek medical care for their FA have a more impaired HRQL and perceive their FA as more severe. Food avoidance and issues related to the EI of FA are key areas of intervention aimed at improving HRQL in patients with FA.

\section{Acknowledgements}

This work was funded by the EU through EuroPrevall (FP6FOOD-CT-2005-514000).

\section{References}

$>1$ Rona RJ, Keil T, Summers C, Gislason D, Zuidmeer L, Sodergren E, et al: The prevalence of food allergy: a meta-analysis. J Allergy Clin Immunol 2007;120:638-646.

-2 Le TM, van Hoffen E, Pasmans SG, BruijnzeelKoomen CA, Knulst AC: Suboptimal management of acute food-allergic reactions by patients, emergency departments and general practitioners. Allergy 2009;64:1227-1228.

3 King RM, Knibb RC, Hourihane JO: Impact of peanut allergy on quality of life, stress and anxiety in the family. Allergy 2009;64:461468.

4 Flokstra-de Blok BM, Dubois AE, Vlieg-Boerstra BJ, Oude ElberinkJN, Raat H, et al: Healthrelated quality of life of food allergic patients: comparison with the general population and other diseases. Allergy 2010;65:238-244.

5 Marklund B, Ahlstedt S, Nordstrom G: Health-related quality of life in food hypersensitive schoolchildren and their families: parents' perceptions. Health Qual Life Outcomes 2006;4:48.
6 Mills EN, Mackie AR, Burney P, Beyer K, Frewer L, Madsen C, et al: The prevalence, cost and basis of food allergy across Europe. Allergy 2007;62:717-722.

7 Kummeling I, Mills EN, Clausen M, Dubakiene R, Perez CF, Fernandez-Rivas M, et al: The EuroPrevall surveys on the prevalence of food allergies in children and adults: background and study methodology. Allergy 2009;64: 1493-1497.

$>8$ Flokstra-de Blok BM, van der Meulen GN, DunnGalvin A, Vlieg-Boerstra BJ, Oude Elberink JN, Duiverman EJ, et al: Development and validation of the Food Allergy Quality of Life Questionnaire - Adult Form. Allergy 2009;64:1209-1217.

$>9$ Mueller HL: Diagnosis and treatment of insect sensitivity. J Asthma Res 1966;3:331-333.

10 Peeters KA, Nordlee JA, Penninks AH, Chen L, Goodman RE, Bruijnzeel-Koomen CA, et al: Lupine allergy: not simply cross-reactivity with peanut or soy. J Allergy Clin Immunol 2007; 120:647-653.

11 Jaeschke R, Singer J, Guyatt GH: Measurement of health status: ascertaining the minimal clinically important difference. Control Clin Trials 1989;10:407-415.
12 van der Velde JL, Flokstra-de Blok BM, VliegBoerstra BJ, Oude Elberink JN, Schouten JP, DunnGalvin A, et al: Test-retest reliability of the Food Allergy Quality of Life Questionnaires (FAQLQ) for children, adolescents and adults. Qual Life Res 2009;18:245-251.

13 Sampson HA, Mendelson L, Rosen JP: Fatal and near-fatal anaphylactic reactions to food in children and adolescents. N Engl J Med 1992;327:380-384.

14 Cherepanov D, Palta M, Fryback DG, Robert SA: Gender differences in health-related quality-of-life are partly explained by sociodemographic and socioeconomic variation between adult men and women in the US: evidence from four US nationally representative data sets. Qual Life Res 2010;19:1115-1124.

15 Ellert U, Kurth BM: Health-related quality of life in adults in Germany: results of the German Health Interview and Examination Survey for Adults (DEGS1) (in German). Bundesgesundheitsblatt Gesundheitsforschung Gesundheitsschutz 2013;56:643-649. 